\title{
El MIR de Medicina Física y Rehabilitación, la especialidad y el Espacio Europeo de Educación Superior: impresiones desde la universidad
}

\author{
Residency in Physical Medicine and Rehabilitation, the specialty and European Space \\ Of Upper Education: impressions from the university
}

S. Santos del Riego

Desde las Declaraciones de La Sorbona (1998) y Bolonia (1999) hasta la Declaración de Budapest/Viena (2010), se ha ido conformando el Espacio Europeo de Educación Superior (EEES) con la incorporación, participación e implantación progresiva en 46 países europeos. Esta iniciativa se amplía a América Latina y Caribe (Espacio UEALC), en un modelo general de organización convergente inspirado en algunos aspectos de la universidad norteamericana. Se configura un sistema oficial de estudios (Grado, Máster Universitario y Doctorado) comprensible, comparable y transparente, que emplea como herramientas políticas de calidad, consenso, documentación y rendición de cuentas. El EEES permite la diversidad competitiva de títulos en una misma disciplina, favorece el empleo, la movilidad y el intercambio de estudiantes, profesores y profesionales, promociona un modelo de aprendizaje a lo largo de la vida (life-long learning) y establece un área europea de investigación 1. En España, el RD 1393/2007, de 29 de Octubre, por el que se establece la ordenación de las enseñanzas universitarias oficiales, transpone y detalla los principios de esta reforma en títulos de Grados y Másteres universitarios, indicando que en el presente curso 2010-2011 sólo se podrán ofertar plazas de nuevo ingreso para estos títulos ${ }^{2}$.

De forma similar a la adaptación de los títulos universitarios al EEES, el Ministerio de Sanidad y Consumo, a través de la Comisión Nacional de Especialidades, ha comenzado con el proceso de convergencia europea de la sanidad española. En este contexto, se ha planteado un sistema renovado de formación especializada de Médicos Internos Residentes (MIR) basado en la definición de competencias comunes a varias especialidades, agrupándolas en diversas troncalidades durante un tiempo determinado de su periodo formativo. Una vez se concrete este sistema, los programas de formación de cada especialidad desarrollarán una cultura convergente en los hospitales y centros de salud españoles, en sintonía con Europa y con la reforma universitaria efectuada. Se pretende mejorar el rol profesional con una formación teórico-clínica completa del especialista médico, con perfiles innovadores ajustados al conocimiento científico-técnico y a la realidad sociolaboral, facilitando la resolución de aspectos comunes entre especialidades, favoreciendo la adaptación del especialista a entornos sanitarios, sociales y sociosanitarios, contribuyendo a la coordinación en los equipos interdisciplinarios, potenciando el paradigma social de salud frente al paradigma clásico y promoviendo la movilidad del médico residente y del especialista en Europa ${ }^{3}$ and 4 . Aunque en un primer momento se planteó no participar de la troncalidad en el sistema MIR renovado, desde la Comisión Nacional de la especialidad, la Sociedad Española de Rehabilitación y Medicina Física (SERMEF) y la Asociación de Profesores Universitarios de Medicina Física y Rehabilitación (APUMEFYR), se cerró un debate colectivo con la propuesta de inclusión de la especialidad en la troncalidad médica, a efectos de potenciar el rol médico del especialista en Medicina Física y Rehabilitación (MFR) como elemento diferenciador de otras profesiones afines, entre otros propósitos. Surge la oportunidad para reflexionar, reconsiderar y redefinir objetivos - lo que se pretende desde la perspectiva del tutor de residentes-, competencias — uso integrado de conocimientos, habilidades y actitudes - y resultados de aprendizaje — lo que el especialista médico en formación será capaz de demostrar a posteriori- ${ }^{5}$ and 6 . Conviene pensar sobre las competencias genéricas 0 transversales que se deben adquirir y potenciar, como la capacidad para trabajar en equipo, y obliga a redefinir las competencias específicas de cada especialidad que, conjuntamente, determinarán en las 
próximas décadas el ámbito de formación y el rol competencial en un marco de gran competitividad profesional e interprofesional ${ }^{6}$. La Unión Europea de Médicos Especialistas (UEMS) ha elaborado los requisitos de los programas de formación de las diferentes especialidades médicas, el currículo, las competencias técnicas y los procedimientos mínimos diagnósticos y terapéuticos que acreditará el MIR para obtener el certificado de la especialidad ${ }^{7}$. El primer libro blanco de la UEMS ha sido el Libro Blanco de MFR, especialidad existente en 21 de 25 países europeos ${ }^{7}$. En España, la Orden SCO/846/2008, de 14 de marzo, por la que se aprueba y publica el programa formativo de la especialidad de Medicina Física y Rehabilitación, explicita la doctrina vigente que se enseña/aprende en nuestra especialidad ${ }^{8}$. Sería prioritario e interesante profundizar en los programas de formación específicos de MFR y en las disposiciones legales profesionales de países europeos y de otros continentes (Estados Unidos/Canadá/Australia).

Las reformas «globalizadoras» europeas obligarán a superar los compartimentos estancos que existen entre la formación universitaria (Grado de Medicina), la realización de las enseñanzas de Doctorado, la Formación Especializada MIR y el desarrollo profesional continuo. Todo apunta a participaciones más intensas entre complejos hospitalarios y universidades, con los hospitales - y otras estructuras sanitarias, incluso sociosanitarias - llamados a convertirse en el corazón de las nuevas enseñanzas de Medicina y de otras Ciencias de la Salud, sean universitarias o sanitarias, en ámbitos asistenciales, docentes, investigadores, de innovación biomédica, transferencia de conocimiento, formación continuada y de organización ${ }^{9}$. Las barreras deben superarse, integrándose la gestión entre las instituciones sanitarias y la universidad, asumiendo conjuntamente y de forma unificada la docencia de la rama de Ciencias de la Salud. A modo de ejemplo, se ha implantado algún innovador título de Grado en Medicina compartido entre varias universidades, con un número reducido de estudiantes, que centran su actividad en el ámbito hospitalario (Universidad Pompeu Fabra/Universidad Autónoma de Barcelona [UAB]/Hospital del Mar), otros priman en algunas materias el aprendizaje interprofesional de estudiantes de varias titulaciones afines, con metodologías como el aprendizaje basado en problemas (ABP) u ofrecen asignaturas optativas en itinerarios dispuestos para orientar al estudiante a la diversa troncalidad que se pretende establecer en la formación especializada ${ }^{9}$. Incluso, autores como Rozman $(2005)^{10}$ proponen crear Universidades de Ciencias de la Salud como modelo integrador del sistema sanitario y el universitario a efectos de superar y solventar el tradicional desentendimiento y descoordinación entre los ministerios de Sanidad y de Educación.

En este contexto de creatividad innovadora, las recomendaciones que desde la universidad y desde APUMEFYR hacemos a los MIR en particular y al resto de los profesionales de la especialidad en general, a efectos de potenciar tanto el curriculum vitae individual como la proyección colectiva de la especialidad de MFR, son: $a$ ) realizar las nuevas enseñanzas de doctorado, que se concretarán en una tesis doctoral, y b) participar como profesores en materias afines de Rehabilitación en las diversas titulaciones de grado de la rama de Ciencias de la Salud (Medicina, Terapia Ocupacional, Fisioterapia, Enfermería y Logopedia, entre otras), másteres universitarios oficiales y enseñanzas de doctorado y otros títulos propios (cursos de formación específica de posgrado, cursos de especialistas — expertos- y másteres clásicos), teniendo que acreditarse previamente para algunas de las diversas figuras de profesorado en la Agencia Nacional de Evaluación de la Calidad y Acreditación (ANECA) o en alguna agencia de calidad universitaria autonómica.

En relación con la realización de la tesis doctoral, conviene reflexionar, de forma similar a los modelos establecidos en Italia o Francia, si todo especialista médico debería concluir su formación MIR con la realización de una tesis doctoral como condición sine qua non previa al ejercicio asistencial, docente, investigador y gestor de su especialidad. Es lícito conocer que el RD 99/2011, de 28 de enero, por el que se regulan las enseñanzas oficiales de Doctorado, refiere en el artículo 6.b los requisitos de acceso, que permiten el paso directo del graduado en Medicina al doctorado sin necesidad de realizar un máster universitario ${ }^{11}$. Asimismo, de la lectura del apartado 6.c se infiere la posibilidad de acceder directamente a las enseñanzas de doctorado a los MIR de tercer año y siguientes. Aunque no suele ser grato plantear estudios universitarios simultáneos con la formación MIR, dada la plétora de actividades asistenciales, se considerará que el nivel de doctorado en profesiones afines se está incrementando notablemente y, en el marco del EEES, loablemente. Los hospitales universitarios se tendrían que adaptar a los nuevos condicionantes y demandas asistenciales, investigadoras y docentes de las enseñanzas universitarias de grado y doctorado en Medicina, a efectos de incluir la realización de una tesis doctoral, o al menos el inicio previsto en el RD 99/2011, de forma adecuada y adaptada a la realidad asistencial del MIR.

El desarrollo de un perfil completo del especialista se enriquecerá con la participación como docente/investigador en la universidad, impartiendo en diversas titulaciones y potenciando la especialidad de MFR en otros ámbitos significativos de toma de decisiones. Las posibilidades más factibles son como tutor docente-asistencial con venia docendi o, mejor aún, como profesor asociado. Las plazas de profesor 
asociado se proponen desde el consejo de departamento pertinente y son aprobadas por el consejo de gobierno de cada universidad, para profesionales específicos de reconocido prestigio, resolviéndose por un tribunal conformado por profesores universitarios de áreas afines, una vez establecidos los perfiles y criterios para cada plaza y realizado el baremo. Para el acceso a las figuras de profesor universitario contratado (a saber: profesor de universidad privada, profesor ayudante doctor, profesor colaborador y profesor contratado doctor), hará falta la acreditación positiva de la ANECA (o agencia de calidad universitaria autonómica), según los condicionantes que se exponen en el Programa de Evaluación del Profesorado (PEP) ${ }^{12}$. Se evalúa la formación académica, la experiencia docente, investigadora y profesional y otros méritos del interesado ${ }^{12,13}$ and 14 . A posteriori, si hubiera necesidad institucional y oportunidad personal, el candidato acreditado podrá presentarse al concurso de alguna de estas plazas ofertadas en la universidad ${ }^{12,13 \text { and } 14}$. Para el acceso a los cuerpos de funcionarios docentes universitarios, tal es el caso de profesores titulares de universidad (TU) y catedráticos de universidad (CU), se establece en el Programa ACADEMIA una fase de acreditación nacional realizada en la ANECA (o agencia de calidad universitaria autonómica) y, de resultar positiva, posteriormente se realiza el pertinente concurso a plazas específicas ofertadas en las universidades ${ }^{15}$. Para la acreditación nacional de estas figuras, se valora la formación (sólo en TU) y la experiencia docente (o profesional), investigadora, de gestión y administración ${ }^{13,14}$ and 15 . A posteriori, en los concursos que se realizan en la universidad, los candidatos exponen el curriculum vitae junto con los proyectos docente, investigador $\mathrm{y}$, en plaza vinculada, asistencial ${ }^{13,14}$ and 15

La especialidad de MFR no se puede anclar en modelos estáticos del pasado y debe rediseñarse en parámetros innovadores y europeos. La implementación del nuevo sistema MIR obligaría a redefinir un programa de formación competitivo que permita establecer un modelo profesional autónomo, desde el respeto y participación en la interdisciplinariedad. Es altamente positivo que la SERMEF y sus filiales hayan establecido grupos de trabajo, que abordan y replantean las derivas que nos aporta la modernidad, tales como la troncalidad, los modelos de actuación, el marco competencial autónomo y convergente de la especialidad, la interacción en el equipo interdisciplinario, superando modelos caducos de comunicación e interrelación, entre otros. La MFR será lo que todos y cada uno de sus profesionales, con compromiso colectivo, trabajo y dedicación quieran que sea.

\section{Bibliografía}

1. L. Christensen. The Bologna process and medical education. Med Tech, 26 (2004), pp. 525-529.

2. Real Decreto 1393/2007, de 29 de Octubre, por el que se establece la ordenación de las enseñanzas universitarias oficiales, Ministerio de Educación y Ciencia. Boletín Oficial del Estado, núm. 260, de 30-10-2007.

3. Consejo Nacional de Especialidades en Ciencias de la Salud, Ministerio de Sanidad y Consumo. Ministerio de Ciencia e Innovación. La Troncalidad en las Especialidades Médicas 2008. Madrid: Ministerio de Sanidad y Consumo; 2008. Disponible en:

http://www.aeemt.com/contenidos_socios/actividades/CNEMT_Contenidos/DOCUMENTO_TRONCALIDAD_ version_01_01.pdf.

4. Orden ECI/332/2008, de 13 de febrero, por la que se establecen los requisitos para la verificación de los Títulos universitarios oficiales que habiliten para el ejercicio de la profesión de médico. Ministerio de Educación y Ciencia. Boletín Oficial del Estado, núm. 40, de 15-2-2008.

5. Guía para la evaluación de competencias en el Trabajo de Fin de Grado en el ámbito de las Ciencias Sociales y Jurídicas. Barcelona: Agència per a la Qualitat del Sistema Universitari de Catalunya; 2005.

6. García San Pedro MJ, Gairín Sallán J. Los mapas de competencias: Una herramienta para mejorar la calidad de la formación universitaria. Revista Iberoamericana sobre Calidad, Eficacia y Cambio en Educación. 2011; 9.

7. Libro Blanco de Medicina Física y Rehabilitación [versión en inglés]. Mediterranean Journal of Physical and Rehabilitation Medicine. 2006; 42.

8. Orden SCO/846/2008, de 14 de marzo, por la que se aprueba y publica el programa formativo de la especialidad de Medicina Física y Rehabilitación, Ministerio de Educación y Ciencia. Boletín Oficial del Estado, núm. 77, de 293-2008.

9. J. Millán Núñez-Cortés, J.A. Gutiérrez Fuentes. Directores. Educación Médica, 13 Suppl 1 (2010).

10. C. Rozman, J.L. Lafarga-Traver. La Universidad de Ciencias de la Salud: aspectos legales de una nueva opción. Med Clin (Barc), 124 (2005), pp. 499-503.

11. Real Decreto 99/2011, de 28 de enero, por el que se regulan las enseñanzas oficiales de Doctorado, Ministerio de Educación y Ciencia. Boletín Oficial del Estado, núm. 35, de 10-2-2011.

12. Programa de Evaluación del Profesorado (PEP), Agencia Nacional de Evaluación de la Calidad y Acreditación (ANECA). Disponible en: http://www.aneca.es/Programas/PEP.

13. Real Decreto 1312/2007, de 5 de octubre, por el que se establece la acreditación nacional para el acceso a los cuerpos docentes universitarios, Ministerio de Educación y Ciencia. Boletín Oficial del Estado, núm. 240, de 610-2007. 
14. Real Decreto 1313/2007, de 5 de octubre, por el que se regula el régimen de los concursos de acceso a los cuerpos docentes universitarios, Ministerio de Educación y Ciencia. Boletín Oficial del Estado, núm. 241, de 8-10-2007.

15. Programa ACADEMIA, Agencia Nacional de Evaluación de la Calidad y Acreditación (ANECA). Disponible en: http://www.aneca.es/Programas/ACADEMIA. 